\title{
A Case of Pemphigus Vulgaris with Serum Autoantibodies Binding Blood Cell Antigen(s) on Immunofluorescence: A Case Report
}

\author{
Alessandro Lanza ${ }^{1,2}$, Felice Femiano ${ }^{1,2}$, Giovanni Maria Gaeta ${ }^{1}$, Rossella Santoro ${ }^{2}$, \\ Fernando Gombos ${ }^{1,2}$ and Nicola Cirillo ${ }^{*, 1,2,3}$
}

\author{
${ }^{1}$ Regional Center on Craniofacial Malformations-MRI, ${ }^{2}$ Department of Odontostomatology, ${ }^{3}$ Department of \\ Experimental Medicine, $1^{\text {st }}$ School of Medicine and Surgery, II University of Naples, 80138 Naples, Italy
}

\begin{abstract}
Patients with pemphigus vulgaris (PV) may develop autoimmunity against an array of non-desmoglein antigens, including keratinocyte acetylcholine receptors. The latter, along with yet unknown autoantigens, could participate to PV pathogenesis. Here, we report a case of PV developing autoimmunity against peripheral blood mononuclear cell (PBMC) antigens. The patient was poorly responsive to steroidal and immunosuppressive drugs. Although the role for this PBMC antigen has not been established, it could affect the behaviour of immune cells that regulate autoantibody production in PV. Deregulation of PBMC activity may therefore influence patient's response to pharmacological therapy.
\end{abstract}

\section{INTRODUCTION}

Pemphigus represents a group of autoimmune blistering diseases characterized by the loss of cell-cell adhesion between keratinocytes (acantholysis). Indeed, both types of pemphigus, vulgaris (PV) and foliaceus (PF), primarily affect stratified epithelia, such as skin and mucous membranes [1]. Consistently, the main autoantigens of pemphigus are cell adhesion proteins occurring in the desmosome, namely desmoglein (Dsg) 1 and Dsg3. In addition, non-desmoglein antigens expressed by keratinocytes may contribute to the early signaling events leading to acantholysis [2]. Recently, however, the discovery of a circulating $30 \mathrm{kDa}$ fragment of desmoglein $3(\mathrm{sDsg} 3)$ has moved the attention from tissuerestricted epithelial antigens to those found in the serum [3]. This led us to the discovery that PV IgG isolated from four patients were able to immunoprecipitate a $130 \mathrm{kDa}$ antigen expressed by peripheral blood mononuclear (PBMC) cells [4]. By means of immunofluorescence analysis, here we describe the fifth PV patient with circulating antibodies against PBMC.

\section{CASE PRESENTATION}

A 41-year-old male came to the Stomatology Clinic of the Second University of Naples with a 6-month history of bullous disorder. The patient initially went to community dentist and received topical steroids for 3 months without a precise diagnosis being established. Topical treatment was then discontinued and the patient was subsequently referred to our clinic. He complained sore mouth and difficulty in eating. On clinical examination, he showed painful erosions involving gums, cheek, tongue, palate, and labial mucosa (Fig. 1). Skin involvement was limited, with three small bullae on his chest (not shown). The clinical picture was suggestive of PV. The results of serologic analysis showed positive titres of anti-intercellular substance (ICS) IgG by means of indirect immunofluorescence (IIF) on monkey oesophagus, thus confirming the diagnosis of PV (Table 1). Immuno-

*Address correspondence to this author at the Department of Odontostomatology, Second University of Naples, Via Luigi de Crecchio, 7, 80138 Naples, Italy; E-mail: cirillo.sun@libero.it reactivity of PV IgG was further identified by immunoprecipitation of $\mathrm{HaCaT}$ extracts with PV sera [5]. Western blot analysis of the immunocomplexes revealed that PV IgG recognized both Dsg3 and Dsg1 (Table 1). This datum seems apparently in contrast with the current knowledge, as autoantibodies in mucosal dominant PV are expected to react only with Dsg3. However, the presence of anti-Dsg1 IgG does not necessarily correlate with gross skin involvement.
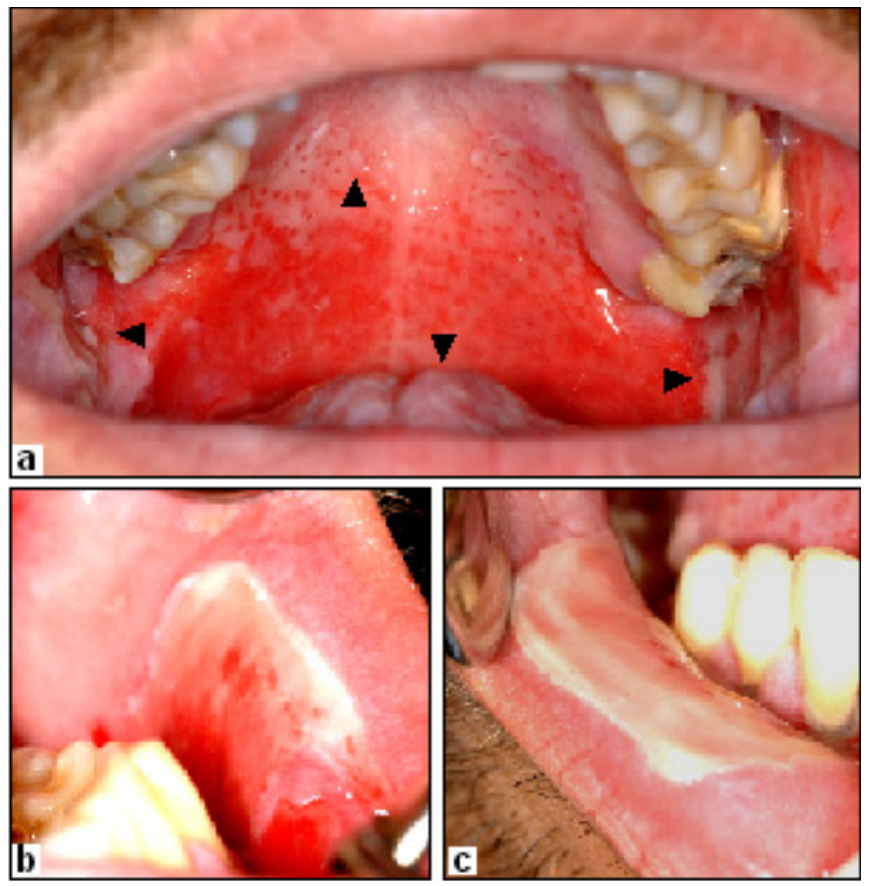

Fig. (1). Involvement of the oral mucosa. Typical erosions with irregular margins covered by whitish pseudomembranes on tongue, buccal mucosa (bilaterally), and palate (a, arrowheads). Lesions also affected labial mucosa (b,c).

The patient was commenced on high doses systemic steroids (90 mg/day deflazacort) plus immunosuppressive agents (azathioprine $100 \mathrm{mg} /$ day). However, he did not display good responsiveness to such a dose of steroids, that was accordingly escalated to $150 \mathrm{mg}$ /day deflazacort for three 
weeks. The dose of steroids was then tapered to $120 \mathrm{mg} /$ day. One month later, the patient returned for clinical control and serum sample was taken.

Table 1. Findings of the Indirect Immunofluorescence (IIF) and Western Blotting (WB) Analyses On Patient's Serum

\begin{tabular}{|c|c|c|}
\hline \multirow{2}{*}{ IIF } & \multicolumn{2}{|c|}{$1: 160$} \\
\cline { 2 - 3 } & anti-Dsg3 IgG & anti-Dsg1 IgG \\
\hline WB & + & + \\
\hline
\end{tabular}

Since PV autoimmunity seems to be not just restricted to epidermal antigens, the serum sample was used to test the immunoreactivity of patient's IgG to blood cell antigens by immunofluorescence microscopy, following the procedure reported by us elsewhere [4]. PBMC were collected from two volunteers without any skin disease and incubated with PV serum. Immunofluorescence microscopy on PBMC revealed that PV IgG recognized antigen(s) located on cell surface. Indeed, FITC fluorescence was localized all around cell membrane (Fig. 2). On the contrary, none of the controls displayed any pericellular staining of $\operatorname{IgG}$ (Fig. 2a). The slight fluorescence in control experiments may be due to unspecific binding of IgG to PBMC during immunofluorescence procedures.
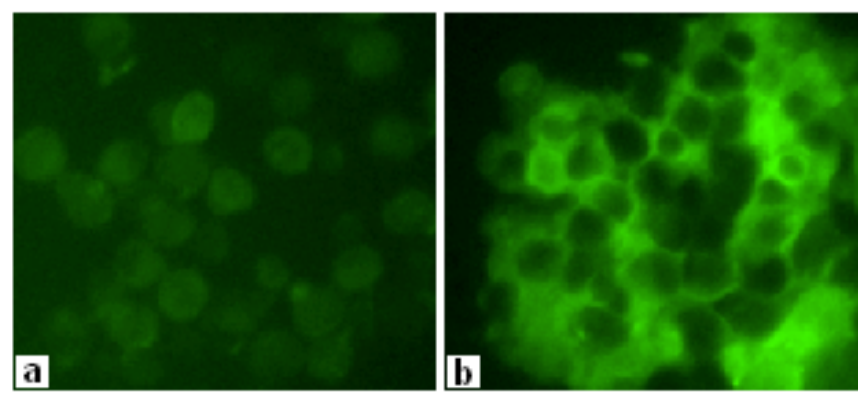

Fig. (2). Immunofluorescence analysis reveals that $\mathrm{IgG}$ from pemphigus vulgaris (PV) (b), but not normal (a) sera bind surface of peripheral blood mononuclear cells (PBMC). Non-specific background of FITC fluorescence was detectable in controls (a).

Collectively, these data demonstrate that our patient developed autoimmunity against PBMC.

\section{COMMENTS}

A number of studies have shown that PV IgG fraction contains an array of autoantibodies against different keratinocyte antigens, ranging from 12 to $190 \mathrm{kD}$ (reviewed in ref. [6]). By indirect immunofluorescence, these antibodies produce a typical "fishnet-like" pattern in Dsg3-knockout mice [7]. In addition to adhesion molecules, such as Dsg1, Dsg3, desmocollins, plakoglobin, E-cadherin, and collagen XVII/BP180, a list of known targets for pemphigus antibodies also includes receptor molecules, such as annexins, and AChRs (reviewed in ref [8]). We have recently discovered a $130 \mathrm{kD}$ antigen other than Dsg3, which has been immunoprecipitated with PV IgG from PBMC [4]. It is reasonable that the molecule on PBMC surface recognized by PV IgG in this study is the same as described previously, but the lack of immunoprecipitation analysis does not allow to address this question. Overall, these novel findings have fostered an alternative concept on the pathophysiological relevance of non-desmoglein targets in PV known as "multiple hit" hypothesis [9].

Current concepts strongly suggest that autoreactive $T$ cells play a crucial role in the initiation and perpetuation of both umoral and cell-mediated autoimmune diseases [10]. Autoreactive T cells may provide critical help for B cells to continuously produce pathogenic autoantibodies in PV. Consistently, Sugiyama and colleagues reported the proportion of Treg cells in PV patients to be severely reduced, approximately ten times less than controls [11]. Accordingly, one can speculate that PV IgG against PBMC may affect the Treg compartment, thus promoting the activity of autoreactive T lymphocytes. In this regard, it is worth of note that novel therapies for PV patients refractory to steroideal treatment rely on the action of lymphocyte-targeting antibodies, such as rituximab [12].

In conclusion, we have described a case of PV carrying circulating autoantibodies against PBMC antigens. Although their biological role still need to be understood, PBMCtargeting autoantibodies may affect lymphocyte function and regulation of the immune response.

\section{REFERENCES}

[1] Bystryn JC, Rudolph JL. Pemphigus. Lancet 2005; 366: 61-73.

[2] Chernyavsky AI, Arredondo J, Kitajima Y, Sato-Nagai M, Grando SA. Desmoglein $v s$ non-desmoglein signaling in pemphigus acantholysis: Characterization of novel signaling pathways downstream of pemphigus vulgaris antigens. J Biol Chem 2007; 282: 13804-12.

[3] Lanza A, Femiano F, De Rosa A, Cammarota M, Lanza M, Cirillo $\mathrm{N}$. The N-terminal fraction of desmoglein 3 encompassing its immunodominant domain is present in human serum: implications for pemphigus vulgaris autoimmunity. Int J Immunopathol Pharmacol 19: 399-407.

[4] Cirillo N, Gombos F, Lanza A. Pemphigus vulgaris immunoglobulin $\mathrm{G}$ can recognize a 130,000 MW antigen other than desmoglein 3 on peripheral blood mononuclear cell surface. Immunology 2007; 121: 377-82.

[5] Cirillo N, Femiano F, Gombos F, Lanza A. Serum from pemphigus vulgaris reduces desmoglein 3 half-life and perturbs its de novo assembly to desmosomal sites in cultured keratinocytes. FEBS Lett 2006; 580: 3276-81.

[6] Lanza A, Cirillo N, Femiano F, Gombos F. How does acantholysis occur in pemphigus vulgaris: a critical review. J Cutan Pathol 2006; 33: 401-12.

[7] Nguyen VT, Ndoye A, Shultz LD, Pittelkow MR, Grando SA. Antibodies against keratinocyte antigens other than desmogleins 1 and 3 can induce pemphigus vulgaris-like lesions. J Clin Invest 2000; 106: 1467-79.

[8] Grando SA. Autoimmunity to keratinocyte acetylcholine receptors in pemphigus. Dermatology 2000; 201: 290-5

[9] Grando SA. Pemphigus in the XXI Century: new life to an old story. Autoimmunity 2006; 39: 521-30.

[10] Hertl M, Eming R, Veldman C. T cell control in autoimmune bullous skin disorders. J Clin Invest 2006; 116:1159-66.

[11] Sugiyama H, Matsue H, Nagasaka A, et al. CD4+CD25high regulatory $\mathrm{T}$ cells are markedly decreased in blood of patients with pemphigus vulgaris. Dermatology 2007; 214: 210-20.

[12] Ahmed AR, Spigelman Z, Cavacini LA, Posner MR. Treatment of pemphigus vulgaris with rituximab and intravenous immune globulin. N Engl J Med 2006; 355: 1772-9. 